KYUNGPOOK Math. J. 55(2015), 83-89

http://dx.doi.org/10.5666/KMJ.2015.55.1.83

pISSN 1225-6951 eISSN 0454-8124

(c) Kyungpook Mathematical Journal

\title{
Convolution on a Generalized Class of Harmonic Univalent Functions
}

\author{
SAURABH PorWaL* \\ Department of Mathematics, U. I. E. T. Campus, C. S. J. M. University, Kanpur- \\ 208024 (U. P.), India \\ e-mail : saurabhjcb@rediffmail.com
}

Kaushal Kishore Dixit

Department of Engineering Mathematics Gwalior Institute of Information Technology, Gwalior-474015, (M. P.), India

e-mail : kk.dixit@rediffmail.com

Abstract. In the present paper, we introduce new subclasses of harmonic univalent functions and establish certain results concerning the convolution of functions for these subclasses. Relevant connections of the results presented here with various known results are briefly indicated.

\section{Introduction}

A continuous complex-valued function $f=u+i v$ is said to be harmonic in a simply connected domain $D$ if both $u$ and $v$ are real harmonic in $D$. In any simply connected domain we can write $f=h+\bar{g}$, where $h$ and $g$ are analytic in $D$. We call $h$ the analytic part and $g$ the co-analytic part of $f$. A necessary and sufficient condition for $f$ to be locally univalent and sense-preserving in $D$ is that $\left|h^{\prime}(z)\right|>\left|g^{\prime}(z)\right|, z \in D$, (see Clunie and Sheil-Small [4]). For more basic results on harmonic functions one may refer to the following standard text book by Duren [8], see also Ahuja [1] and Ponnusamy and Rasila ([14], [15]).

Denote by $S_{H}$ the class of functions $f=h+\bar{g}$ which are harmonic univalent and sense-preserving in the open unit disk $U=\{z:|z|<1\}$ for which $f(0)=$ $f_{z}(0)-1=0$. Then for $f=h+\bar{g} \in S_{H}$ we may express the analytic functions $h$ and $g$ as

* Corresponding Author.

Received July 27, 2012; accepted April 24, 2013.

2010 Mathematics Subject Classification: 30C45, 26D05.

Key words and phrases: Analytic, Univalent, Harmonic Functions, Convolution. 


$$
h(z)=z+\sum_{k=2}^{\infty} a_{k} z^{k}, \quad g(z)=\sum_{k=1}^{\infty} b_{k} z^{k}, \quad\left|b_{1}\right|<1 .
$$

Note that $S_{H}$ reduces to the class $S$ of normalized analytic univalent functions if the co-analytic part of its member is zero. A function $f$ of the form (1.1) is in the class $S_{H}^{*}$, if it satisfies

$$
\frac{\partial}{\partial \theta}\left\{\arg f\left(r e^{i \theta}\right)\right\} \geq 0, \quad\left\{z=r e^{i \theta} \in U\right\},
$$

and is in the class $K_{H}$, if and only if

$$
\frac{\partial}{\partial \theta}\left\{\arg \left(\frac{\partial}{\partial \theta} f\left(r e^{i \theta}\right)\right)\right\}>0, \quad\left\{z=r e^{i \theta} \in U\right\} .
$$

The classes $S_{H}^{*}$ and $K_{H}$ respectively consisting of functions harmonic starlike and harmonic convex in $U$, have been studied by Silverman [16], Silverman and Silvia [17], (see also Avci and Zlotkiewicz [3] and Jahangiri [11]).

Also, Öztürk and Yalcin [13] investigated two new subclasses $H S(\alpha),(0 \leq \alpha<$ $1)$ and $H C(\alpha),(0 \leq \alpha<1)$ of harmonic starlike and convex functions respectively.

A function $f(z)$ defined by (1.1) is said to be in the class $H S(\alpha),(0 \leq \alpha<1)$ if and only if

$$
\sum_{k=2}^{\infty}\left(\frac{k-\alpha}{1-\alpha}\right)\left(\left|a_{k}\right|+\left|b_{k}\right|\right) \leq\left(1-\left|b_{1}\right|\right)
$$

and in the class $H C(\alpha),(0 \leq \alpha<1)$ if and only if

$$
\sum_{k=2}^{\infty} \frac{k(k-\alpha)}{1-\alpha}\left(\left|a_{k}\right|+\left|b_{k}\right|\right) \leq\left(1-\left|b_{1}\right|\right) .
$$

Recently, Dixit and Porwal [7] generalized these classes $H S(\alpha)$ and $H C(\alpha)$ to the class $H S(m, n, \alpha)$.

A function $f(z)$ defined by (1.1) is said to be in the class $H S(m, n, \alpha)$, if and only if

$$
\sum_{k=2}^{\infty} \frac{k^{m}-\alpha k^{n}}{1-\alpha}\left(\left|a_{k}\right|+\left|b_{k}\right|\right) \leq\left(1-\left|b_{1}\right|\right),
$$

where $m \in N, n \in N_{0}, m>n, 0 \leq \alpha<1$.

It should be worthy to note that by specializing the parameter in $H S(m, n, \alpha)$, we obtain the following known subclasses which have been studied by various researchers.

1. The classes $H S(1,0, \alpha) \equiv H S(\alpha)$ and $H S(2,1, \alpha) \equiv H C(\alpha)$ were studied by Öztürk and Yalcin [13]. 
2. The classes $H S(1,0,0) \equiv H S$ and $H S(2,1,0) \equiv H C$ were studied by Avci and Zlotkiewicz [3].

For $b_{1}=0$, the classes $H S(\alpha), H C(\alpha)$ and $H S(m, n, \alpha)$ are denoted by $H S^{0}(\alpha)$, $H C^{0}(\alpha)$ and $H S^{0}(m, n, \alpha)$ respectively.

Let $\phi(z)$ be a fixed function of the form

$$
\phi(z)=z+\sum_{k=2}^{\infty} c_{k} z^{k}, \quad\left(c_{k} \geq c_{2}>0, \quad k \geq 2\right) .
$$

Now, using the function $\phi(z)$ we define the following new subclasses of $S_{H}$.

Definition 1.1. A function $f(z) \in M_{H}^{0}\left(c_{k}, \delta\right),\left(c_{k} \geq c_{2}>0 ; k \geq 2\right)$ if and only if

$$
\sum_{k=2}^{\infty} c_{k}\left(\left|a_{k}\right|+\left|b_{k}\right|\right) \leq \delta, \quad(\delta>0) .
$$

Definition 1.2. A function $f(z) \in N_{H}^{0}\left(c_{k}, \delta\right),\left(c_{k} \geq c_{2}>0 ; k \geq 2\right)$ if and only if

$$
\sum_{k=2}^{\infty} k c_{k}\left(\left|a_{k}\right|+\left|b_{k}\right|\right) \leq \delta, \quad(\delta>0) .
$$

Definition 1.3. A function $f(z) \in B_{H}^{n}\left(c_{k}, \delta\right),\left(c_{k} \geq c_{2}>0 ; k \geq 2\right)$ if and only if

$$
\sum_{k=2}^{\infty} k^{n} c_{k}\left(\left|a_{k}\right|+\left|b_{k}\right|\right) \leq \delta
$$

where $\delta>0$ and $n$ is any fixed nonnegative real number.

It is worthy to note that if $g \equiv 0$ the classes $M_{H}^{0}\left(c_{k}, \delta\right), N_{H}^{0}\left(c_{k}, \delta\right)$ and $B_{H}^{0}\left(c_{k}, \delta\right)$ reduce to the classes $M_{s}^{0}\left(c_{k}, \delta\right), N_{s}^{0}\left(c_{k}, \delta\right)$ and $B_{s}^{0}\left(c_{k}, \delta\right)$, respectively, studied by Frasin and Aouf in [10].

It is easy to see that various subclasses of harmonic univalent functions as well as analytic univalent functions can be represented as $B_{H}^{n}\left(c_{k}, \delta\right)$ for suitable choices of $c_{k}, \delta$ and $n$ studied by various authors. For example:

1. $B_{H}^{0}(k, 1) \equiv S_{H}^{*} \quad($ Silverman $[16])$

2. $B_{H}^{0}\left(k^{2}, 1\right) \equiv H C \quad($ Avci and Zlotkiweicz [3])

3. $B_{H}^{0}(k-\alpha, 1-\alpha) \equiv H S(\alpha),(0 \leq \alpha<1) \quad$ (Öztürk and Yalcin [13])

4. $B_{H}^{0}(k(k-\alpha), 1-\alpha) \equiv H C(\alpha),(0 \leq \alpha<1) \quad$ (Öztürk and Yalcin [13])

5. $B_{H}^{0}\left(k^{m}-\alpha k^{n}, 1-\alpha\right) \equiv H S(m, n, \alpha),\left(m \in N, n \in N_{0}, m>n, 0 \leq \alpha<1\right)$ (Dixit and Porwal [7]) 
Evidently, $B_{H}^{0}\left(c_{k}, \delta\right) \equiv M_{H}^{0}\left(c_{k} \delta\right)$ and $B_{H}^{1}\left(c_{k}, \delta\right) \equiv N_{H}^{0}\left(c_{k}, \delta\right)$. Further,

$$
B_{H}^{n}\left(c_{k}, \delta\right) \subset B_{H}^{h}\left(c_{k}, \delta\right), \quad \text { if } \quad n>h \geq 0 .
$$

To prove our main result we need the following definition of convolution.

For harmonic functions of the form

$$
f(z)=z+\sum_{k=2}^{\infty} a_{k} z^{k}+\sum_{k=1}^{\infty} \overline{b_{k} z^{k}}
$$

and

$$
F(z)=z+\sum_{k=2}^{\infty} A_{k} z^{k}+\sum_{k=1}^{\infty} \overline{B_{k} z^{k}}
$$

we define the convolution

$$
(f * F)(z)=f(z) * F(z)=z+\sum_{k=2}^{\infty} a_{k} A_{k} z^{k}+\sum_{k=1}^{\infty} \overline{b_{k} B_{k} z^{k}} .
$$

Similarly, we can define the convolution of more than two functions.

Several authors such as ([2], [5], [6], [9], [10], [12]) studied the convolution properties of analytic univalent functions only, yet analogues results on harmonic univalent functions have not been explored in great detail so far in the literature. In this paper, an attempt has been made to systematically study on convolution of functions in the classes $B_{H}^{n}\left(c_{k}, \delta\right), M_{H}^{0}\left(c_{k}, \delta\right)$ and $N_{H}^{0}\left(c_{k}, \delta\right)$.

Throughout this paper, we assume the functions $f(z), g(z), f_{i}(z) a n d g_{j}(z)$ in the following form

$$
\begin{gathered}
f(z)=z+\sum_{k=2}^{\infty} a_{k} z^{k}+\sum_{k=1}^{\infty} \overline{b_{k} z^{k}}, \\
g(z)=z+\sum_{k=2}^{\infty} d_{k} z^{k}+\sum_{k=1}^{\infty} \overline{e_{k} z^{k}}, \\
f_{i}(z)=z+\sum_{k=2}^{\infty} a_{k, i} z^{k}+\sum_{k=1}^{\infty} \overline{b_{k, i} z^{k}}, \quad(i=1,2, \ldots, m)
\end{gathered}
$$

and

$$
g_{j}(z)=z+\sum_{k=2}^{\infty} d_{k, j} z^{k}+\sum_{k=1}^{\infty} \overline{e_{k, j} z^{k}}, \quad(j=1,2, \ldots, q) .
$$




\section{Main Results}

Theorem 2.1. Let the functions $f_{i}(z)$ defined by (1.8) with $b_{1, i}=0$ for every $i=1,2, \ldots m$ belong to the class $N_{H}^{0}\left(c_{k}, \delta\right)$ and let the functions $g_{j}(z)$ defined by (1.9) with $e_{1, j}=0$ for every $j=1,2, \ldots, q$ be in the class $M_{H}^{0}\left(c_{k}, \delta\right)$. If $c_{k} \geq k \delta$, then the convolution $f_{1} * f_{2} * \ldots * f_{m} * g_{1} * g_{2} * \ldots * g_{q}(z)$ belongs to the class $B_{H}^{2 m+q-1}\left(c_{k}, \delta\right)$.

Proof. Let

$$
\begin{aligned}
h(z) & =f_{1} * f_{2} * \ldots * f_{m} * g_{1} * g_{2} * \ldots * g_{q}(z) \\
& =z+\sum_{k=2}^{\infty}\left\{\Pi_{i=1}^{m} a_{k, i} \Pi_{j=1}^{q} d_{k, j}\right\} z^{k}+\sum_{k=1}^{\infty} \overline{\left\{\Pi_{i=1}^{m} b_{k, i} \Pi_{j=1}^{q} e_{k, j}\right\} z^{k}} .
\end{aligned}
$$

We have to show that

$$
\sum_{k=2}^{\infty}\left[k^{2 m+q-1} c_{k}\left(\left\{\Pi_{i=1}^{m} a_{k, i} \Pi_{j=1}^{q} d_{k, j}\right\}+\left\{\Pi_{i=1}^{m} b_{k, i} \Pi_{j=1}^{q} e_{k, j}\right\}\right)\right] \leq \delta .
$$

Since $f_{i}(z) \in N_{H}^{0}\left(c_{k}, \delta\right)$, we have

$$
\sum_{k=2}^{\infty} k c_{k}\left(\left|a_{k, i}\right|+\left|b_{k, i}\right|\right) \leq \delta
$$

for every $i=1,2, \ldots, m$. Therefore

$$
\left|a_{k, i}\right| \leq \frac{\delta}{k c_{k}} \quad \text { and } \quad\left|b_{k, i}\right| \leq \frac{\delta}{k c_{k}}
$$

which implies that

$$
\left|a_{k, i}\right| \leq \frac{1}{k^{2}} \quad \text { and } \quad\left|b_{k, i}\right| \leq \frac{1}{k^{2}}
$$

for every $i=1,2, \ldots, m$.

Similarly, for $g_{j}(z) \in M_{H}^{0}\left(c_{k}, \delta\right)$, we have

$$
\sum_{k=2}^{\infty} c_{k}\left(\left|d_{k, j}\right|+\left|e_{k, j}\right|\right) \leq \delta
$$

for every $j=1,2, \ldots, q$. Hence we obtain

$$
\left|d_{k, j}\right| \leq \frac{1}{k} \quad \text { and } \quad\left|e_{k, j}\right| \leq \frac{1}{k},
$$

for every $j=1,2, \ldots, q$. 
Now, using $(2.4)$ for $i=1,2, \ldots, m,(2.6)$ for $j=1,2, \ldots, q-1$ we obtain

$$
\begin{aligned}
& \sum_{k=2}^{\infty}\left[k^{2 m+q-1} c_{k}\left(\left\{\Pi_{i=1}^{m}\left|a_{k, i}\right| \Pi_{j=1}^{q}\left|d_{k, j}\right|\right\}+\left\{\Pi_{i=1}^{m}\left|b_{k, i}\right| \Pi_{j=1}^{q}\left|e_{k, j}\right|\right\}\right)\right] \\
\leq & \sum_{k=2}^{\infty}\left[k^{2 m+q-1} c_{k}\left(\frac{1}{k^{2 m}} \frac{1}{k^{q-1}}\left|d_{k, q}\right|+\frac{1}{k^{2 m}} \frac{1}{k^{q-1}}\left|e_{k, q}\right|\right)\right] \\
= & \sum_{k=2}^{\infty} c_{k}\left(\left|d_{k, q}\right|+\left|e_{k, q}\right|\right) \quad[\mathrm{Using}(2.5) \text { for } j=q] \\
\leq & \delta .
\end{aligned}
$$

Therefore $h(z) \in B_{H}^{2 m+q-1}\left(c_{k}, \delta\right)$.

Thus the proof of Theorem is established.

Taking into account the convolution of functions $f_{1}(z), f_{2}(z), \ldots, f_{m}(z)$ only, in the proof of above theorem, and using (2.4) for $i=1,2, \ldots, m-1$ and (2.3) for $i=m$, we obtain the following corollary.

Corollary 2.2. Let the functions $f_{i}(z)$ be defined by (1.8) with $b_{1, i}=0, \quad(i=$ $1,2,3, \ldots$.$) be in the class N_{H}^{0}\left(c_{k}, \delta\right)$ for every $i=1,2, \ldots, m$. Then the convolution $f_{1} * f_{2} * \ldots * f_{m}$ belongs to the class $B_{H}^{2 m-1}\left(c_{k}, \delta\right)$.

Next, taking into account the convolution of functions $g_{1}(z),\left(g_{2}(z), \ldots, g_{q}(z)\right.$ only, in the proof of the above theorem, and using (2.6) for $j=1,2, \ldots, q-1$ and (2.5) for $j=q$, we obtain the following corollary.

Corollary 2.3. Let the functions $g_{j}(z)$ be defined by (1.9) with $e_{1, j}=0, \quad(j=$ $1,2,3, \ldots, q)$ be in the class $M_{H}^{0}\left(c_{k}, \delta\right)$ for every $j=1,2, \ldots, q$. Then the convolution $g_{1} * g_{2} * \ldots * g_{g}(z)$ belongs to the class $B_{H}^{q-1}\left(c_{k}, \delta\right)$.

Remark 1. If the co-analytic part of $f_{i}(z)$ and $g_{j}(z)$ are zero for every $i=1,2, \ldots, m$ and $j=1,2, \ldots, q$. Then we obtain the corresponding result given by Frasin and Aouf in [10].

Remark 2. Taking $c_{k}=k-\alpha$ and $\delta=1-\alpha,(0 \leq \alpha<1)$ with co-analytic part zero in the above theorem, we obtain the main result given by Kumar in [12].

Remark 3. Taking $c_{k}=(1-\beta) k-\alpha \beta$ and $\delta=\beta(1-\alpha),(0 \leq \alpha<1,0 \leq \beta<1 / 2)$ with co-analytic part zero in the above theorem, we obtain the main result given by Darwish in [5].

Remark 4. Taking $c_{k}=(k-1)+\beta(1+k \alpha)$ and $\delta=\beta(1+\alpha),(0 \leq \alpha<1,0 \leq \beta<1)$ with co-analytic part zero in the above theorem, we obtain the main result given by Aouf in [2].

Remark 5. Taking $c_{k}=k(1+m)-(m+\alpha)$ and $\delta=(1-\alpha),(0 \leq \alpha<1,0 \leq$ $m<\infty)$ with co-analytic part zero in the above theorem, we obtain the main result given by Frasin in [9]. 
Acknowledgements. The authors are thankful to the referee for his/her valuable comments and suggestions.

\section{References}

[1] O. P. Ahuja, Planar harmonic univalent and related mappings, J. Inequal. Pure Appl. Math., 6(4)(2005), Art. 122, 1-18.

[2] M. K. Aouf, The quasi-Hadamard product of certain analytic functions, Appl. Math. Lett., 21(11)(2008), 1184-1187.

[3] Y. Avci and E. Zlotkiewicz, On harmonic univalent mappings, Ann. Univ. Mariae Curie-Sklodowska Sect., A 44(1990), 1-7.

[4] J. Clunie and T. Sheil-Small, Harmonic univalent functions, Ann. Acad. Sci. Fen. Series A. I. Math., 9(1984), 3-25.

[5] E. W. Darwish, The quasi-hadamard product of certain starlike and convex functions, Appl. Math. Lett., 20(6)(2007), 692-695.

[6] K. K. Dixit and Vikas Chandra, Quasi-hadamard product of certain uniformly convex and corresponding starlike functions, News Bull. Cal. Math. Soc., 28(1-3)(2005), 1924 .

[7] K. K. Dixit and Saurabh Porwal, On a subclass of harmonic univalent functions, J. Inequal. Pure Appl. Math., 10(1)(2009), Art. 27, 1-18.

[8] P. Duren, Harmonic mappings in the plane, Camb. Univ. Press, 2004.

[9] B. A. Frasin, Quasi-hadamard product of certain classes of uniformly analytic functions, General Math., 16(2)(2007), 29-35.

[10] B. A. Frasin and M. K. Aouf, Quasi-hadamard product of generalized class of analytic and univalent functions, Appl. Math. Lett., 23(4)(2010), 347-350.

[11] J. M. Jahangiri, Harmonic functions starlike in the unit disk, J. Math. Anal. Appl., 235(1999), 470-477.

[12] Vinod Kumar, Quasi-hadamard product of certain univalent functions, J. Math. Anal. Appl., 126(1987), 70-77.

[13] M. Öztürk and S. Yalcin, On univalent harmonic functions, J. Inequal. Pure Appl. Math., 3(4)(2002), Art. 61, 1-8.

[14] S. Ponnusamy and A. Rasila, Planar harmonic mappings, RMS Mathematics Newsletters, 17(2)(2007), 40-57.

[15] S. Ponnusamy and A. Rasila, Planar harmonic and quasi-conformal mappings, RMS Mathematics Newsletters, 17(3)(2007), 85-101.

[16] H. Silverman, Harmonic univalent functions with negative coefficients, J. Math. Anal. Appl., 220(1998), 283-289.

[17] H. Silverman and E. M. Silvia, Subclasses of harmonic univalent functions, New Zealand J. Math., 28(1999), 275-284. 